\title{
Research on Enterprise Strategic Decisions in Big Data Era
}

\author{
Pan Mei \\ School of Economics and Management \\ Tongji University \\ Shanghai, 200000, China
}

\begin{abstract}
Enterprise managers must formulate correct strategic decisions rapidly because of the increasing complexity of management environment. The article analyzed the concept connotation and the development of big data. Integrating real cases, the article also analyzed how big data providerich data sources for enterprises'strategic decisions, and how it can improve the quality of strategic decision making. It also demonstrated the support function of big data in upgrading strategic decision makers'insights. Finally the article proposed some problems and relevant suggestions of big data during its developing process.
\end{abstract}

Keywords-big data; enterprise strategic

\section{INTRODUCTION}

Strategic decision is an extremely important part of strategic management. It determines the success or failure of the enterprise management and is related to the survival and development of the enterprises.Fast strategic decisions which only rely on the decision maker's knowledge, experience, intuition, judgment, personal preferences and other subjective behavior in dynamic, uncertain environment is not enough. They must also rely on data resourceswhichcome from the enterprise external. Data is the foundation of all the management decision making. Making the data analysis to achieve in-depth understanding of customers and enhance the competitiveness of enterprises based on. Today's world has entered the age of big data. Big data provides a new choice for enterprise strategic decision.

\section{CONCEPT OF BIG DATA}

Big data is a relatively abstract concept. Currently, the definition of the concept of big data is not uniform. Schonberg known as the "the first man who apply big data in business"points out that the "big" in big data does not refer to the absolute number but the processing mode. We will try to collect comprehensive data, complete data and synthetic data, at the same time, the use of mathematical methods to analyze and its modeling, mining behind the relationship, thus predicting the probability of event happening behind. Tu Zipei, a famous information management expert of our country, believes that the "big" in big data an imaginary reference in his book big data. It refers to the human ability to find large value data contained in the big data. Big data is often equated with the concept of "massive data" and "ultra large scale data" terms of formulation. However, its connotation greatly exceeded the traditional data form, also beyond the processing capacity of the existing techniques. Famous research institutions Gartner consulting company Gartner for large data is defined as: big data refers to the need for new treatment mode can have the stronger decisionmaking ability, insight found strength and process optimization ability of the massive high rates of growth and diversification of information assets. The world famous management consulting company McKinsey’sbig data is defined as the data which cannot use traditional database software tools in a certain period of time, the contents of the acquisition management and processing of data set.

The most representative definitionof many big data definitions is the $3 \mathrm{~V}$ definition. The first $\mathrm{V}$ means volume, namely the data volume is huge; the second $\mathrm{V}$ means variety,namelythere are a lot of data types in big data, such as text, images, audio, video and so on; The third $\mathrm{V}$ means volumevelocity, that is the fast processing speed of big data. In addition, International Data Corporation (IDC) believes that big data should also have the high commercial value. IBM believes big data should be veracity. In short, the definition of big data concept can be based on $3 \mathrm{~V}$ or $4 \mathrm{~V}$ or $5 \mathrm{~V}$.

\section{EFFECTS OF BIG DATA ON ENTERPRISE'S STRATEGIC DECISION}

\section{A: Big Data Can Provide Resource to Help Enterprise's Strategic Decision Making}

Decision content of enterprise strategy management layer is to determine and adjust the business goals and develop the acquisition. These non-structured decision problems not only have big quantity, but also are full of high level of complexity and high difficulty. They directly affect the enterprise's success or failure.This requires strategic decision makers must have a large number of comes from the enterprise external data resources. Therefore, in the course of formulating enterprise decision objective, policymakers from first to last all need data, information collection work. Big data can provide resource to help enterprise's strategic decision making.

Since the 21 stcentury, all kinds data like pictures, text and sound transmission, behind this user habits and the track of the formation of massive data resources, provide a rich source of data for the management of the decision analysis and decision makingwith the rapid development of Internet technology and communication technology. According to the total amount of data statistics in 2013 China produced more than $0.8 Z \mathrm{Z}, 2$ times in total in 2012 is equivalent to the 2009 global data. In 2020 China is expected to total data generated 
will be 201310 times, which more than 8.5ZB. Enterprise data increasing speed is $55 \%$ a year. Data shows that the Chinese outstanding search engine Baidu in 2013 generated the amount of data is close to the EB level. The total amount of data stored in the Alibaba and Tencent is more than 100 $\mathrm{PB}$. The next two to three years there will be more and more industries, large enterprises come into PB 100 PB even EB level data club.

In the age of big data, the enterprises obtain valuable information through the collection and analysis of big data of internal and external. By mining these information, enterprises can forecast market demand, analyze the intelligent decision-making to develop more effective strategies.

\section{B: Big DataCan Promote the Quality of Enterprise's} Strategic Decisions

Business success depends on the correct decision-making strategy. The correct strategy depends on the quality of the data and information.Right data and information can reduce the decision of many uncertain factors. Practice management requires the decision to move towards the scientific, qualitative and quantitative decision to decision combination. The development of big data technology provides the possibility of realizing it. Big data can be thought of as "another expression analysis".It is seeking to extract knowledge from data, and the intelligent activities it into commercial advantage. Big data changes the decision way relying on the experienceof theory and thought for a long time. It helps accurate data analysis replace intuitive judgment, making decision focus back to the questions to itself.

Big data includes not only the large volume, diversity of data set, but also high-speed data acquisition, processing and analysis to the technical architecture to extract value and technology process on the data set. Therefore, the collaborative analysis of big data needs multiple techniques, including cloud computing, visualization, networking, data mining, hadoop technology. For example, social media monitoring platform DataSift successfully predicted the fluctuation of stock price Facebook would delay only a few minutes (less than 20 minutes) through the sentiment orientation analysis on Twitter on the day Facebook listed May 18, 2012. So the real advantage of big data is a collection of statistics and analysis of the intelligent automation of the massive data. Analysis based on large data more global objective and intuitive. Big data is becoming a new research way to assist the managers to make strategic decisions of enterprise.

\section{C: Big DataCan Improve the Insight of Decision Makers}

Data has become one of the most important assets of enterprises in the age of big data. The decisions of enterprises increasingly rely on making analysis on the data, not rely on experience and intuition.Enterprises need to quickly obtain the ability insight into the market and customers from the business data accumulation and information in ubiquitous network. For example, the famous electronic Business Company Amazon design marketing strategies based on a large number of customer data mining.
After the customer purchase of books in the Amazon online bookstore, its sales system will automatically record the bibliography the customer purchasing and browsed. When the customer once again go into the bookstore, the system will recognize his identity, query the customer purchasing and browsing records, analyzesits often buy books category, speculate that the customer's book preferences, and finally recommend the books which can meet the customer's fancy in the bookstore. When the customer buy some books, the system will again record the purchase books category for the customerin order to guide the next recommended bibliography, so move in circles. In this way, the more times the customers go to Amazon Bookstore, the more Amazon will learn about the customers. Amazon will serve customers more professional.

Enterprise can analyze the accumulated large scale data, accurately grasp the consumer interest, preference by using big data technology so as to design a highly accurate, good performance marketing strategy.

\section{CONCLUSIONS AND SUGGESTIONS}

Based on the above analysis, we can see thatbig data provides a new thought and means for enterprise strategic decision. It is no longer empty talk. However, we should also pay attention to the following problems in the development and application of big data.

The first problem is the existing data processing method is most suitable for structured data processing, but the $85 \%$ enterprise existing data is unstructured or semistructured form. Dealing with large amounts of unstructured and semi-structured data is a great challenge to the enterprise itself technology. The second problem is the majority of small and medium-sized enterprises think big data is a technology only big company like Amazon, Facebook, Google, Baidu, Alibaba is concerned. The shortage of large data understanding will make the enterprises lose the opportunity of improve their competitiveness. Small and medium-sized enterprises should realize that big data is not only the game of giants. The small and medium-sized enterprise should seize the opportunity to explore new mode of big data applications to create new value. The last problem is that the big data technology can never replace human creative thinking. The strategic decision rely only on the big data would deviate from the actual situation.

\section{REFERENCE}

[1] ]He Jianhua. An Analysis on the Influence of Big Data on Enterprise Strategic Decisions [J], Contemporary Economic Management, 2014 10

[2] Liang Yajun. Research on Enterprise Strategic Decisions in Big Data Era [J]Economics and Trade Management, 2004, 9

[3] Rainer Schmidt, Michael Möhring. Big Data as Strategic EnablerInsights from Central European Enterprises[J]Business Information Systems, 2014, 4

[4] Vincent Charles, Tatiana Gherman. Achieving Competitive Advantage through Big Data [J] Middle-East Journal of Scientific Research, 2013, 3 